\title{
Crystal Structure of the Zinc-dependent MarR Family Transcriptional Regulator AdcR in the Zn(II)-bound State
}

\author{
Alfredo J. Guerra, Charles E. Dann III, and David P. Giedroc* \\ Department of Chemistry, Indiana University, Bloomington, IN 47405, United States
}

Supporting Information 


\section{Experimental Procedures}

\section{Protein Expression}

\section{Unlabeled Protein}

A pET3a plasmid encoding the $a d c R$ gene was transformed into E. coli BL21(DE3) cells. E. coli cultures were grown in LB medium supplemented with $100 \mu \mathrm{g} / \mathrm{ml}$ ampicillin at $37{ }^{\circ} \mathrm{C}$ with shaking at $250 \mathrm{rpm}$ until reaching an OD600 $=0.6-0.8$. Protein overexpression was induced by isopropyl $\beta$-D-1-thiogalactopyranoside, IPTG, (400 $\mu \mathrm{M}$ final concentration) and the cells allowed to grow overnight at $30^{\circ} \mathrm{C}$ before harvesting.

\section{Se-Met AdcR}

Selenomethionyl AdcR was overexpressed in E. coli by inhibition of the methionine biosynthetic pathway followed by supplementation with selenomethionine as described. ${ }^{1}$ Overexpression was induced by IPTG (400 $\mu \mathrm{M}$ final concentration) followed by growth overnight at $30{ }^{\circ} \mathrm{C}$.

\section{Methyl-Labeled Protein}

E. coli cultures were grown in M9 minimal media prepared in D2O with $\left[{ }^{2} \mathrm{H}_{7},{ }^{13} \mathrm{C}_{6}\right]$-glucose and ${ }^{15} \mathrm{NH}_{4} \mathrm{Cl}$ as the sole carbon and nitrogen sources, respectively. Once the cells reached an OD600 $\approx 0.685 \mathrm{mg}$ of $\alpha-\left[{ }^{13} \mathrm{C}_{5}, 3-{ }^{2} \mathrm{H}_{1}\right]$ ketoisovalerate and $50 \mathrm{mg}$ of $\alpha-\left[{ }^{13} \mathrm{C}_{4}, 3,3-{ }^{2} \mathrm{H}_{2}\right]$ ketoisobutyrate were added to $1 \mathrm{~L}$ culture. Protein overexpression was induced one hour later by IPTG (400 $\mu \mathrm{M}$ final concentration). Cells were allowed to grow overnight before harvesting by low speed centrifugation.

\section{Protein Purification}


AdcR purification was carried out as previously described ${ }^{2}$ with the exception of the selenomethionyl AdcR which was purified in the presence of $10 \mathrm{mM}$ DTT to avoid selenomethionine oxidation.

\section{NMR Experiments}

Methyl group assignments for the apo-, Zn(II)-AdcR and the Zn(II)-AdcR-DNA complex were obtained using a U- $\left[{ }^{2} \mathrm{H},{ }^{15} \mathrm{~N},{ }^{13} \mathrm{C}\right]$ Ile $\delta-\left[{ }^{13} \mathrm{CH}_{3}\right]$ Leu, Val- $\left[{ }^{13} \mathrm{CH}_{3}\left({ }^{13} \mathrm{CH}_{3}\right]\right.$ AdcR (ILV) sample(s) containing only exchangeable backbone and side chain amide protons, $\gamma$-methyl protons of Val residues, and $\delta$-methyl(s) of Ile/Leu residues prepared as described previously ${ }^{3}$. Methyl groups were assigned using ${ }^{1} \mathrm{H},{ }^{13} \mathrm{C} \mathrm{HMQC}$, and HMCM[CG]CBCA experiments. ${ }^{4} \mathrm{NMR}$ spectra for the apoform were collected on a Varian Inova 600 equipped with a room temperature probe at the Texas A\&M University Biomolecular NMR Laboratory. All other NMR spectra were acquired on a Varian DDR $800 \mathrm{MHz}$ spectrometer equipped with a cryogenic probe at the Indiana University MetaCyt Biomolecular NMR laboratory. Typical solution conditions were $200-600 \mu \mathrm{M}{ }^{15} \mathrm{~N}-$

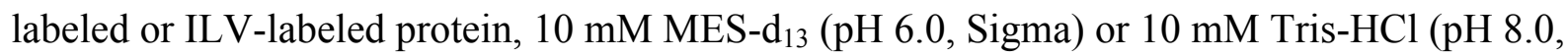
Sigma), $50 \mathrm{mM} \mathrm{NaCl}, 1 \mathrm{mM}$ imidazole, $1 \mathrm{mM}$ TCEP (Gold Biotechnology), 0.02\% (w/v) NaN3

(Fisher) and $10 \%(\mathrm{v} / \mathrm{v}){ }^{2} \mathrm{H}_{2} \mathrm{O}(\mathrm{CIL})$. All spectra were acquired at $35^{\circ} \mathrm{C}$. NMR spectra were processed and analyzed using NMRPipe ${ }^{5}$ and SPARKY ${ }^{6}$. Chemical shift referencing is relative to 2,2-dimethyl-2-silapentene-5-sulfonic acid (DSS; Sigma). ${ }^{7}$

\section{Crystallography}

$\mathrm{Zn}$ (II)-AdcR samples were prepared by adding a slow addition of excess $\mathrm{Zn}$ (II) to apo-AdcR in the presence of $1 \mathrm{mM}$ imidazole. $\mathrm{Zn}$ (II) AdcR and $\mathrm{Zn}$ (II)-SeMet-substituted AdcR were concentrated to $350 \mu \mathrm{M}$ and crystallized in $0.1 \mathrm{M}$ Tris- $\mathrm{HCl}(\mathrm{pH} 8.5), 18 \%$ PEG 10,000, $20 \%$ glycerol, 
and $0.1 \mathrm{M} \mathrm{NaCl}$ (Qiagen) by hanging drop vapor diffusion at $20{ }^{\circ} \mathrm{C}$. Crystals were flash frozen in liquid nitrogen. Intensity data for the native and SeMet-substituted AdcRs were collected at the Advanced Light Source (ALS) Beamline 4.2.2. All data were processed with HKL2000. ${ }^{8}$ Phase calculations were performed by the AutoSol module of PHENIX. ${ }^{9}$ Phases from the SeMet$\mathrm{Zn}(\mathrm{II})-\mathrm{AdcR}$ data set were combined with intensities from the native set in AutoBuild to generate an initial model and electron density maps for the native complex. Model building was performed in Coot $^{10}$ and refinement in PHENIX. 
TABLE S1. Data collection, phasing and refinement statistics for Zn(II)-AdcR

\begin{tabular}{|c|c|}
\hline & $\mathrm{Zn}(\mathrm{II})-\mathrm{AdcR}$ \\
\hline \multicolumn{2}{|l|}{ Data collection } \\
\hline Space group & $\mathrm{P} 22_{1} 2_{1}$ \\
\hline \multicolumn{2}{|l|}{ Cell dimensions } \\
\hline$a, b, c(\AA)$ & $55.848,57.621,85.443$ \\
\hline$\alpha, \beta, \gamma\left({ }^{\circ}\right)$ & $90.00,90.00,90.00$ \\
\hline Resolution $(\AA)$ & $50.00-2.00(2.03-2.00)^{*}$ \\
\hline Rsym & $0.069(0.287)$ \\
\hline$I / \sigma I$ & $21.49(3.80)$ \\
\hline Completeness (\%) & 97 (99.7) \\
\hline Redundancy & $5.7(5.6)$ \\
\hline \multicolumn{2}{|l|}{ Refinement } \\
\hline Resolution $(\AA)$ & 1.998 \\
\hline No. reflections & 17718 \\
\hline$R_{\text {work }} / R_{\text {free }}$ & 0.1847 / 0.2497 \\
\hline \multicolumn{2}{|l|}{ No. atoms } \\
\hline Protein & 2506 \\
\hline Ligand/ion & 4 \\
\hline Water & 302 \\
\hline \multicolumn{2}{|l|}{$B$-factors $\left(\AA^{2}\right)$} \\
\hline Protein & 22.0 \\
\hline Chain A & 18.2 \\
\hline Chain B & 25.6 \\
\hline Ligand/ion & 17.6 \\
\hline Water & 31.0 \\
\hline \multicolumn{2}{|l|}{ R.m.s deviations } \\
\hline Bond lengths $(\AA)$ & 0.004 \\
\hline Bond angles $\left({ }^{\circ}\right)$ & 0.624 \\
\hline
\end{tabular}

*Values in parentheses are for highest-resolution shell 
Table S2. Summary of the Metal binding site geometries for sites 1 and 2

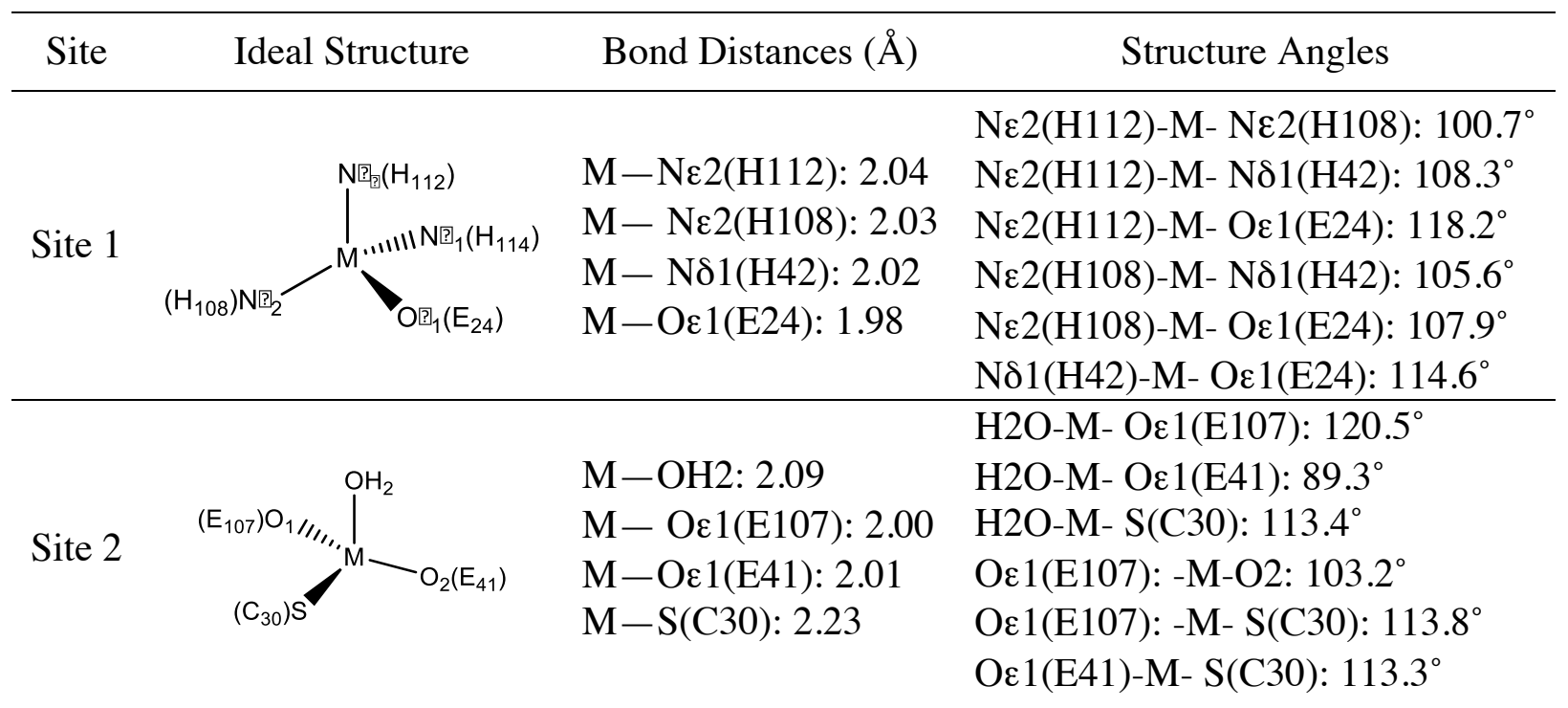


Table S3. Partial residue-specific (non-stereospecific) methyl ${ }^{1} \mathrm{H}$ and ${ }^{13} \mathrm{C}$ chemical shift assignments for the apo-, $\mathrm{Zn}(\mathrm{II})$-bound and DNA-bound ternary AdcR complex. Calculation of the chemical shift perturbation $(\Delta \mathrm{ppm})$ shown in Figure 4 was done using the largest perturbation (in italics) when more than one assignment was available for a particular residue. Overall, 45 of 66 total methyl resonances $(9$ of 10 Ile, 20 of $38 \mathrm{Leu}, 18$ of $20 \mathrm{Val}$ ) were assigned in the apo- form and 56 of 66 total methyl resonances (10 of $10 \mathrm{Ile}, 28$ of $38 \mathrm{Leu}, 19$ of $20 \mathrm{Val}$ ) were assigned in the $\mathrm{Zn}(\mathrm{II})$ - and ternary complex states.

\begin{tabular}{|c|c|c|c|c|c|c|c|c|c|}
\hline \multirow{2}{*}{$\begin{array}{c}\text { Res. } \\
\#\end{array}$} & \multirow[t]{2}{*}{ Assignment } & \multicolumn{2}{|c|}{ Apo-AdcR } & \multicolumn{3}{|c|}{ Zn(II)-AdcR } & \multicolumn{3}{|c|}{ Zn(II)-AdcR-DNA } \\
\hline & & $\begin{array}{c}\delta{ }^{13} \mathrm{C} \\
(\mathrm{ppm})\end{array}$ & $\begin{array}{c}\delta{ }^{1} \mathrm{H} \\
(\mathrm{ppm})\end{array}$ & $\begin{array}{c}\delta{ }^{13} \mathrm{C} \\
(\mathrm{ppm})\end{array}$ & $\begin{array}{c}\delta{ }^{1} \mathrm{H} \\
(\mathrm{ppm})\end{array}$ & $\begin{array}{c}\Delta \text { Sapo- } \\
\mathrm{Zn} \\
(\mathrm{ppm})\end{array}$ & $\begin{array}{c}\delta{ }^{13} \mathrm{C} \\
(\mathrm{ppm})\end{array}$ & $\begin{array}{c}\delta{ }^{1} \mathrm{H} \\
(\mathrm{ppm})\end{array}$ & $\begin{array}{l}\Delta \delta Z n- \\
\text { DNA } \\
\text { (ppm) }\end{array}$ \\
\hline L4 & CD1-MD1 & nd & nd & 22.946 & 0.774 & nd & 22.871 & 0.711 & 0.081 \\
\hline L4 & CD2-MD2 & 22.891 & 0.788 & 23.307 & 0.814 & 1.093 & 23.389 & 0.801 & 0.082 \\
\hline I8 & CD1-MD1 & 14.739 & 0.916 & 14.701 & 0.892 & 0.040 & 14.567 & 0.802 & 0.141 \\
\hline L12 & CD1-MD1 & 23.289 & 0.878 & 23.438 & 0.870 & 1.531 & 23.399 & 0.850 & 0.040 \\
\hline L12 & CD2-MD2 & nd & nd & nd & nd & nd & nd & nd & nd \\
\hline V15 & CG1-MG1 & 22.252 & 1.088 & 23.646 & 1.100 & 1.432 & 23.636 & 1.035 & 0.034 \\
\hline V15 & CG2-MG2 & 20.864 & 1.040 & 20.863 & 1.073 & 0.017 & 20.899 & 1.000 & 0.051 \\
\hline $\mathrm{I} 16$ & CD1-MD1 & 13.160 & 0.773 & 13.181 & 0.553 & 0.112 & 13.217 & 0.502 & 0.044 \\
\hline L17 & CD1-MD1 & nd & nd & 23.372 & 0.943 & nd & nd & nd & nd \\
\hline L17 & CD2-MD2 & nd & nd & 25.150 & 0.978 & nd & 25.247 & 0.889 & 0.107 \\
\hline $\mathrm{I} 25$ & CD1-MD1 & 12.761 & 0.755 & 11.992 & 0.745 & 0.769 & 12.452 & 0.740 & 0.460 \\
\hline L26 & CD1-MD1 & nd & nd & 20.967 & -0.417 & nd & 20.971 & -0.486 & 0.035 \\
\hline L26 & CD2-MD2 & 26.458 & 0.645 & 27.024 & 0.549 & 0.568 & 27.413 & 0.508 & 0.390 \\
\hline $\mathrm{I} 27$ & CD1-MD1 & nd & nd & 10.879 & 0.801 & nd & 10.707 & 0.741 & 0.175 \\
\hline V34 & CG1-MG1 & 20.635 & 0.865 & 20.549 & 0.889 & 0.087 & 20.987 & 0.777 & 0.442 \\
\hline V34 & CG2-MG2 & 20.631 & 0.810 & 21.102 & 0.859 & 0.472 & 21.245 & 0.806 & 0.145 \\
\hline L36 & CD1-MD1 & nd & nd & 27.072 & 0.820 & nd & 26.986 & 0.765 & 0.090 \\
\hline L36 & CD2-MD2 & nd & nd & nd & nd & nd & nd & nd & nd \\
\hline I43 & CD1-MD1 & 15.512 & 0.642 & 14.093 & 0.614 & 1.419 & 13.774 & 0.641 & 0.319 \\
\hline L44 & CD1-MD1 & nd & nd & 25.620 & 0.398 & nd & 25.467 & 0.321 & 0.158 \\
\hline L44 & CD2-MD2 & 19.743 & -0.176 & 19.614 & -0.226 & 0.131 & 19.498 & -0.320 & 0.125 \\
\hline L46 & CD1-MD1 & nd & nd & 22.205 & 0.783 & nd & 22.293 & 0.713 & 0.095 \\
\hline L46 & CD2-MD2 & nd & nd & nd & nd & nd & nd & nd & nd \\
\hline L47 & CD1-MD1 & 23.717 & 1.215 & 23.413 & 1.210 & 0.304 & 23.697 & 1.229 & 0.284 \\
\hline L47 & CD2-MD2 & nd & nd & nd & nd & nd & nd & nd & nd \\
\hline L52 & CD1-MD1 & 23.228 & 0.843 & 23.307 & 0.814 & 0.080 & 23.389 & 0.801 & 0.082 \\
\hline L52 & CD2-MD2 & 26.490 & 0.742 & 26.410 & 0.737 & 0.080 & 26.404 & 0.712 & 0.014 \\
\hline L57 & CD1-MD1 & 25.741 & 0.806 & 25.597 & 0.800 & 0.828 & 25.391 & 0.740 & 0.208 \\
\hline L57 & CD2-MD2 & nd & nd & nd & nd & nd & nd & nd & nd \\
\hline L61 & CD1-MD1 & 23.962 & 0.697 & 23.963 & 0.704 & 0.601 & 23.945 & 0.630 & 0.041 \\
\hline L61 & CD2-MD2 & 25.603 & 0.631 & 25.590 & 0.675 & 0.026 & 25.613 & 0.579 & 0.053 \\
\hline V63 & CG1-MG1 & 19.235 & 0.678 & 19.141 & 0.656 & 0.095 & 18.605 & 0.529 & 0.540 \\
\hline V63 & CG2-MG2 & 21.134 & 0.688 & 20.851 & 0.630 & 0.654 & 20.267 & 0.440 & 0.592 \\
\hline V68 & CG1-MG1 & 22.163 & 0.890 & 21.270 & 0.558 & 0.908 & 21.328 & 0.500 & 0.065 \\
\hline V68 & CG2-MG2 & 24.000 & 0.792 & 23.915 & 0.814 & 0.538 & 23.469 & 0.780 & 0.325 \\
\hline $\mathrm{I} 72$ & CD1-MD1 & 12.898 & 0.188 & 11.614 & 0.286 & 1.285 & 10.694 & -0.158 & 0.946 \\
\hline L75 & CD1-MD1 & 23.363 & 0.754 & 23.222 & 0.736 & 0.713 & 23.264 & 0.670 & 0.053 \\
\hline L75 & CD2-MD2 & nd & nd & nd & nd & nd & nd & nd & nd \\
\hline V76 & CG1-MG1 & 20.864 & 1.040 & 20.865 & 1.027 & 0.011 & 20.746 & 0.965 & 0.123 \\
\hline
\end{tabular}




\begin{tabular}{|c|c|c|c|c|c|c|c|c|c|}
\hline V76 & CG2-MG2 & 22.145 & 0.891 & 22.152 & 0.929 & 0.902 & 22.152 & 0.929 & 0.000 \\
\hline L81 & CD1-MD1 & 24.812 & 0.795 & 24.965 & 0.759 & 0.154 & 24.928 & 0.748 & 0.037 \\
\hline L81 & CD2-MD2 & nd & nd & nd & nd & nd & nd & nd & nd \\
\hline V92 & CG1-MG1 & 20.631 & 0.810 & 20.590 & 0.806 & 0.041 & 20.385 & 0.808 & 0.205 \\
\hline V92 & CG2-MG2 & 21.478 & 0.666 & 21.327 & 0.663 & 0.151 & 21.504 & 0.700 & 0.178 \\
\hline $\mathrm{I} 93$ & CD1-MD1 & 12.243 & 0.730 & 12.391 & 0.717 & 0.148 & 12.007 & 0.672 & 0.385 \\
\hline L97 & CD1-MD1 & 24.740 & 0.866 & 24.777 & 0.836 & 0.010 & 24.530 & 0.795 & 0.248 \\
\hline L97 & CD2-MD2 & nd & nd & 25.411 & 0.826 & nd & 25.411 & 0.826 & 0.000 \\
\hline L100 & CD1-MD1 & 24.036 & 0.944 & 23.959 & 0.963 & 0.078 & 24.014 & 0.896 & 0.064 \\
\hline L100 & CD2-MD2 & nd & nd & 24.103 & 0.907 & nd & 24.014 & 0.896 & 0.089 \\
\hline I104 & CD1-MD1 & 13.361 & 0.894 & 13.940 & 0.959 & 0.580 & 13.839 & 0.885 & 0.108 \\
\hline L116 & CD1-MD1 & 23.228 & 0.843 & 24.149 & 0.851 & 0.921 & 23.950 & 0.836 & 0.199 \\
\hline L116 & CD2-MD2 & 26.431 & 0.773 & 26.329 & 0.773 & 2.619 & 26.324 & 0.750 & 0.013 \\
\hline L117 & CD1-MD1 & 25.349 & 0.740 & 25.149 & 0.730 & 0.200 & 25.071 & 0.658 & 0.086 \\
\hline L117 & CD2-MD2 & nd & nd & nd & nd & nd & nd & nd & nd \\
\hline V122 & CG1-MG1 & 22.802 & 1.149 & 22.799 & 1.131 & 1.291 & 22.835 & 1.065 & 0.049 \\
\hline V122 & CG2-MG2 & 21.509 & 1.021 & 21.460 & 1.011 & 1.344 & 21.389 & 0.932 & 0.081 \\
\hline V133 & CG1-MG1 & 21.511 & 0.786 & 21.576 & 0.780 & 0.422 & 22.007 & 0.726 & 0.432 \\
\hline V133 & CG2-MG2 & 22.832 & 1.236 & 22.938 & 1.236 & 0.150 & 22.943 & 1.160 & 0.038 \\
\hline I134 & CD1-MD1 & 12.172 & -0.416 & 12.130 & -0.437 & 0.043 & 12.048 & -0.518 & 0.091 \\
\hline L138 & CD1-MD1 & nd & nd & nd & nd & nd & nd & nd & nd \\
\hline L138 & CD2-MD2 & nd & nd & nd & nd & nd & nd & nd & nd \\
\hline L141 & CD1-MD1 & 24.740 & 0.866 & 24.579 & 0.883 & 0.161 & 24.551 & 0.839 & 0.036 \\
\hline L141 & CD2-MD2 & 28.285 & 0.705 & 27.187 & 0.702 & 1.098 & 27.045 & 0.738 & 0.143 \\
\hline V142 & CG1-MG1 & 22.253 & 0.465 & 22.490 & 0.354 & 0.438 & 22.738 & 0.256 & 0.253 \\
\hline V142 & CG2-MG2 & 23.082 & 0.974 & 22.803 & 0.931 & 1.931 & 22.803 & 0.931 & 0.000 \\
\hline I145 & CD1-MD1 & 13.387 & 0.805 & 13.750 & 0.728 & 0.365 & 13.261 & 0.758 & 0.489 \\
\hline
\end{tabular}




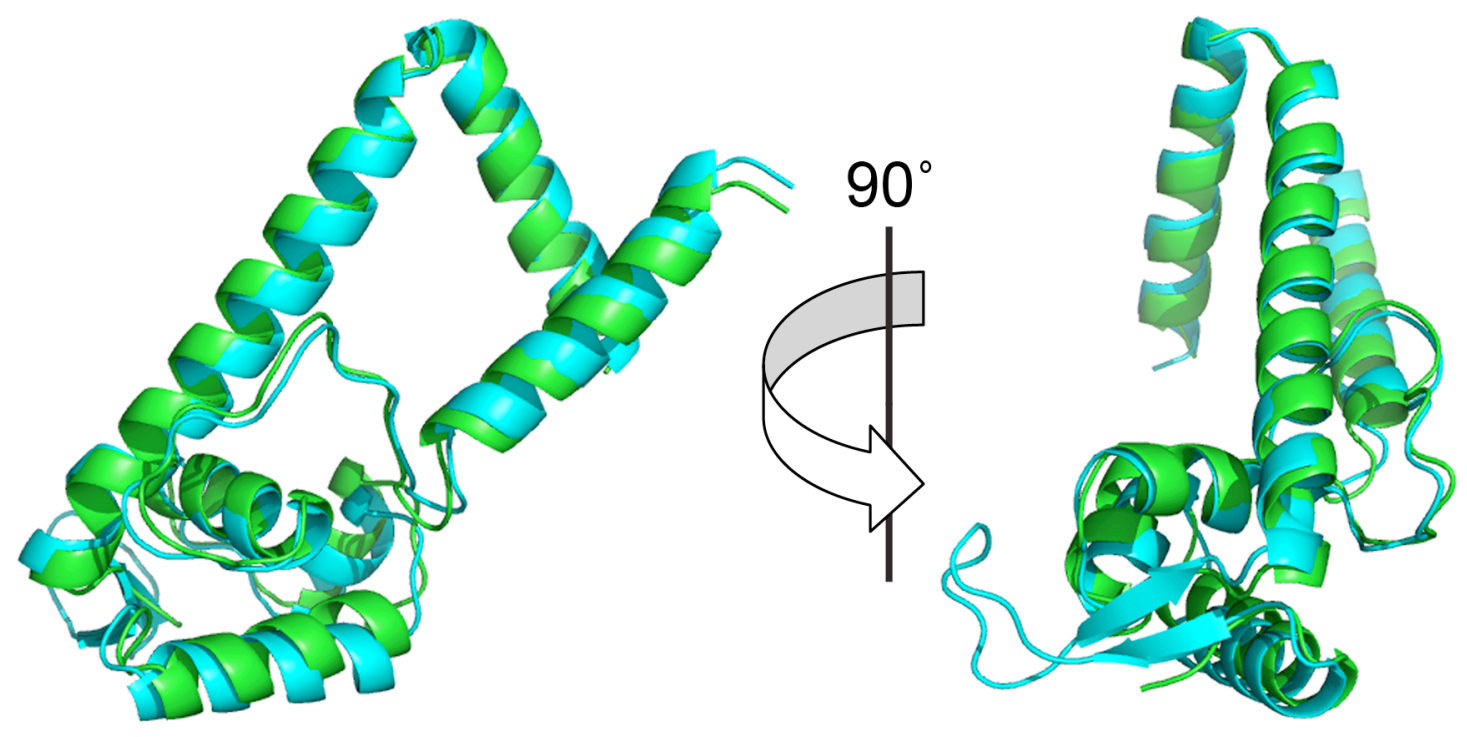

Figure S1. Superimposition of the two AdcR protomers within the homodimer. Chain A is shaded green and chain B is shaded cyan. The two protomers superimpose with a r.m.s.d. of $1.2 \AA$ for the corresponding $129 \mathrm{C} \alpha$ atoms, excluding the $\beta$-wing which is not observed in chain A. 


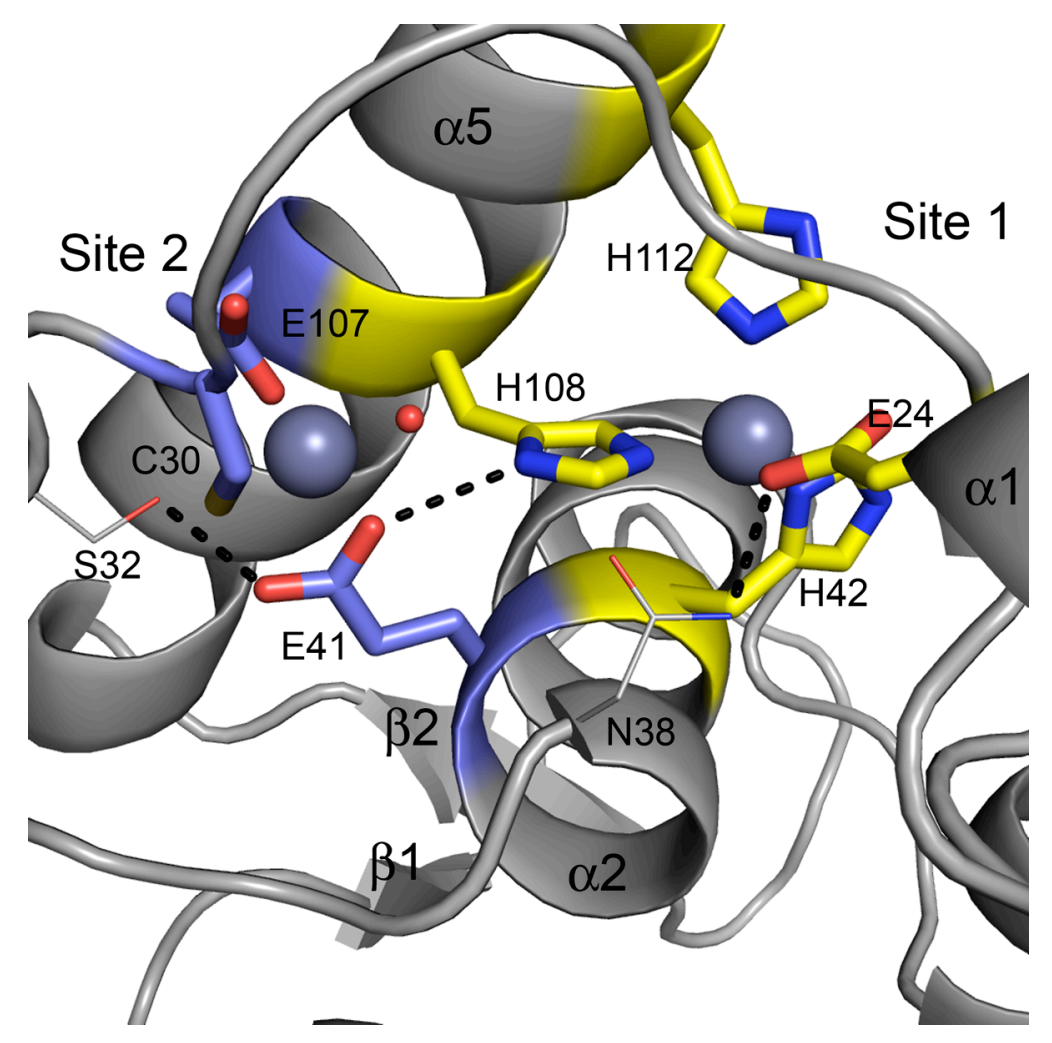

Figure S2. Close up view of sites 1 and 2. Metal binding residues are colored yellow (site 1) and blue (site 2) and highlighted in stick representation. Second coordination sphere residues N38 (zinc site 1) and S32 (zinc site 2) are shown in line representation. Hydrogen bonds are shown as black dashed lines. 

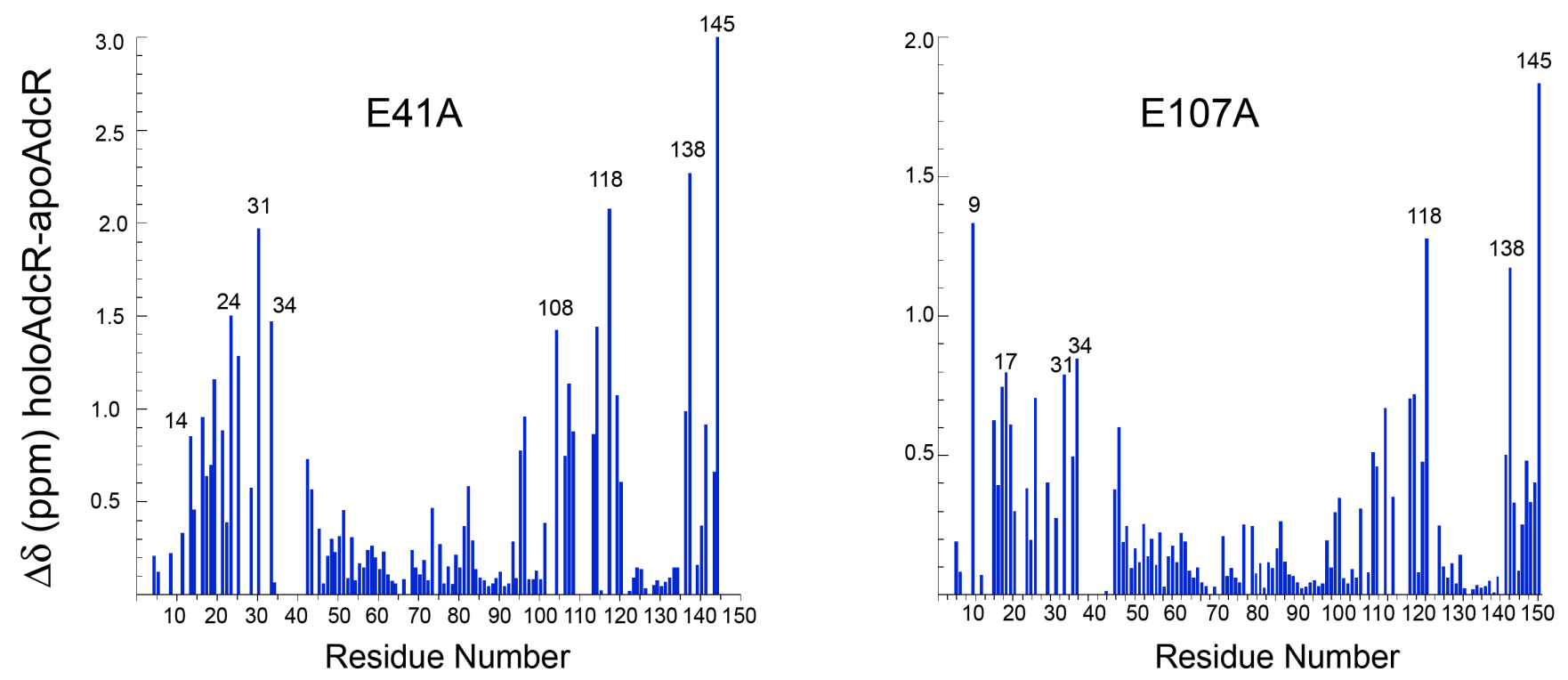

Figure S3. Backbone amide chemical shift perturbation upon $\mathrm{Zn}$ (II) binding to apo-E41A AdcR (A) and apo-E107A AdcR (B) s obtained from analysis of ${ }^{1} \mathrm{H}-{ }^{15} \mathrm{~N}$ TROSY spectra each AdcR with and without 1.0 monomer mol•equiv $\mathrm{Zn}(\mathrm{II})$. Both plots are indicative of large global changes in the spectrum consistent with what is observed in WT-AdcR. ${ }^{2}$ 

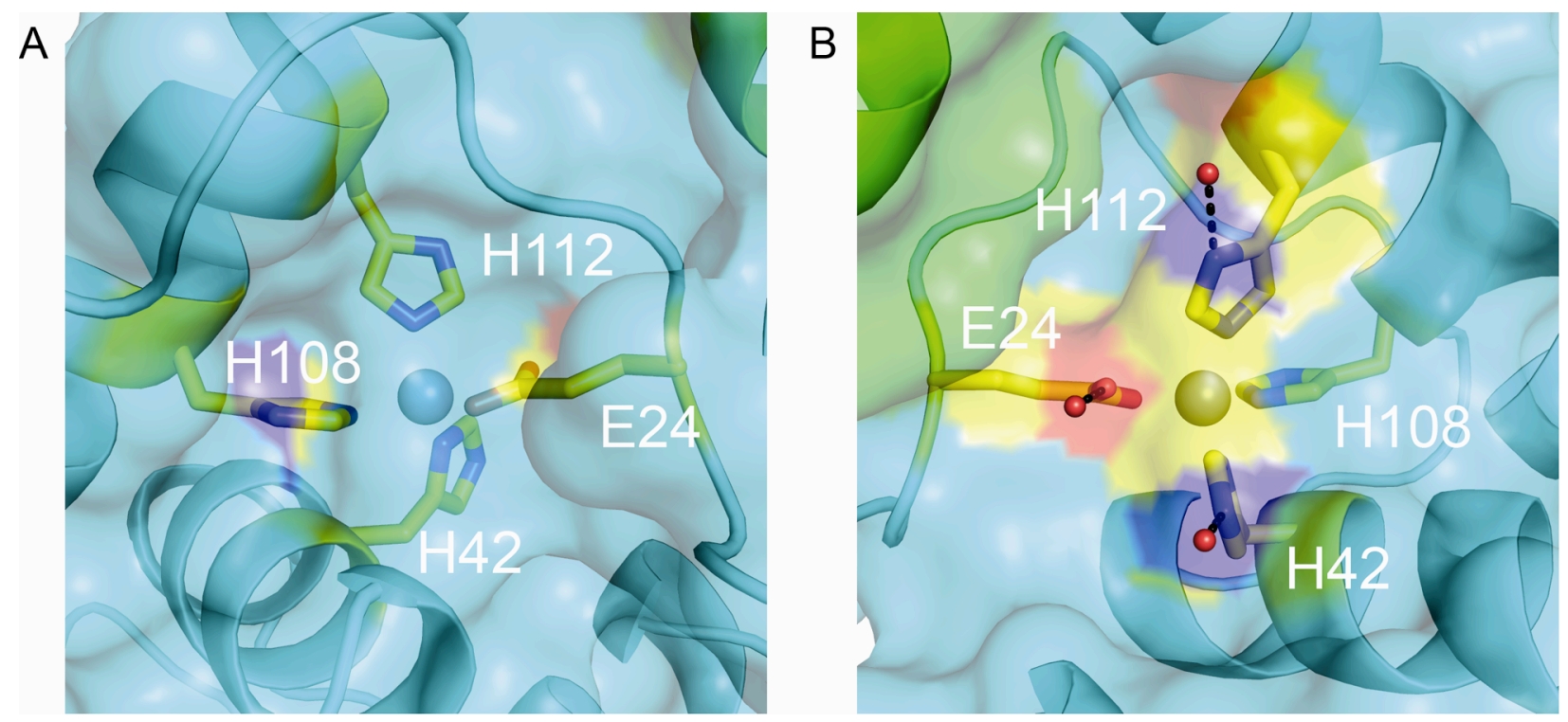

Figure S4. Surface representation of zinc site 1. Metal-binding side chains are highlighted in stick representation with the carbon atoms colored yellow. (A) Orientation is the same as the cyan chain B in Fig. 1. (B) $180^{\circ}$ rotation along the $z$-axis. Both panels illustrate the extent to which site 1 is solvent exposed. The non-liganding Oع2 from E24, Nع2 from $\mathrm{H} 108$ and $\mathrm{N} \delta 1$ from $\mathrm{H} 112$ form hydrogen bonds with water molecules on the surface (see panel B). 


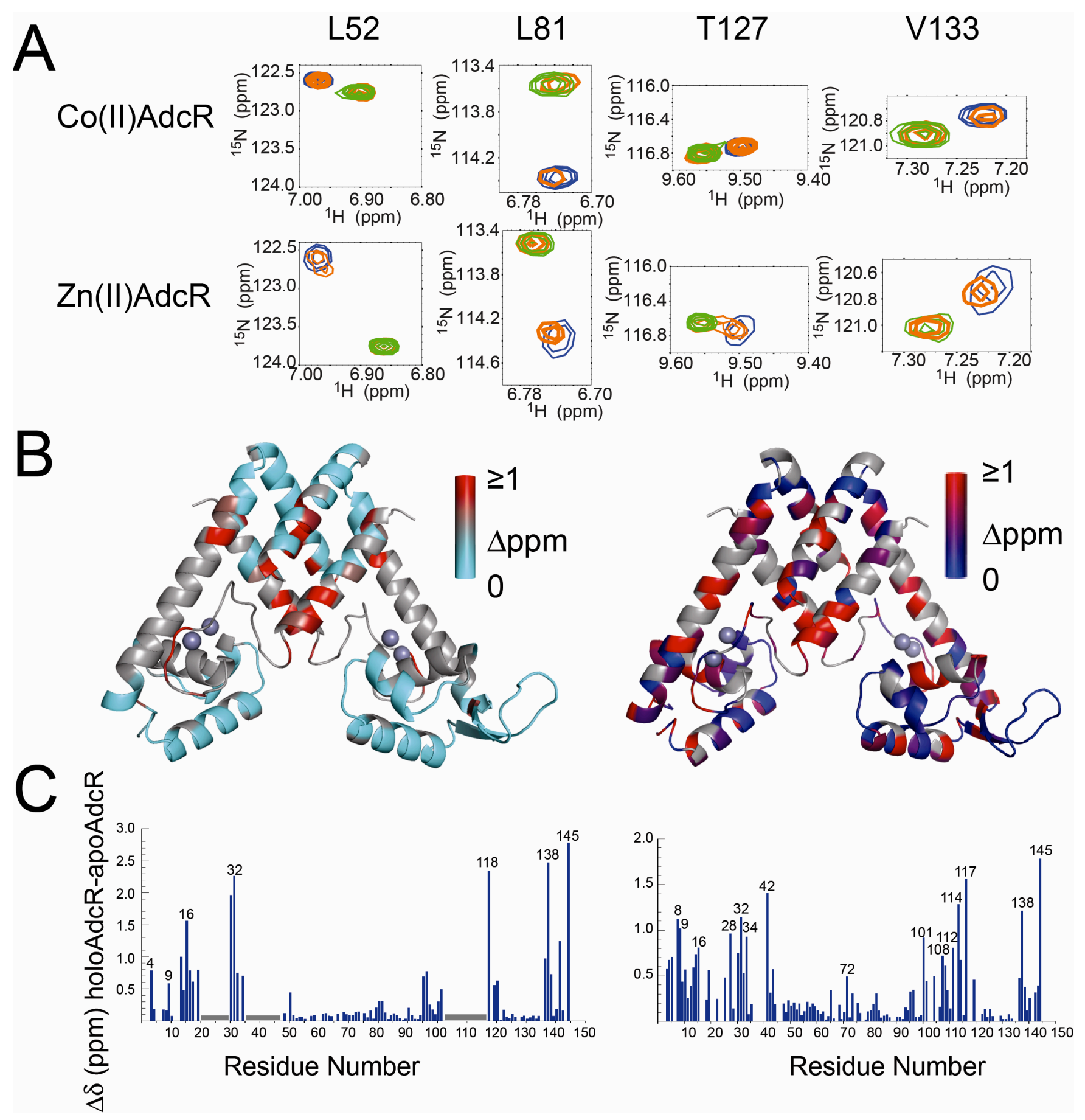

Figure S5. Effect of metal binding on apo AdcR. (A) Co(II) (top) or $\mathrm{Zn}$ (II) (bottom) titration into apoAdcR $\left(\mathrm{pH} 6.0,500 \mu \mathrm{M} \text { AdcR, } K_{\mathrm{Co}}=2.4 \times 10^{5} \mathrm{M}^{-1}\right)^{2}$. Representative crosspeaks (L52, L81, T127, $\mathrm{V} 133$ ) are colored blue for apo-AdcR, orange for 0.5 mol equiv metal added to apo-AdcR, and green for fully metallated AdcR. (B) Backbone amide chemical shift perturbation upon Co(II) (left) and Zn(II) (right) binding to apo-AdcR. Co(II) perturbations are colored from cyan to red and $\mathrm{Zn}$ (II) perturbations are colored from blue to red. Grey residues represent no data, which for Co(II) includes the paramagnetic broadening by $\mathrm{Co}(\mathrm{II})$ at the metal binding site. (C) Backbone amide chemical shift perturbation represented as a bar graph. The grey horizontal bar in the $\mathrm{Co}$ (II) bar graph (left) represents the area broadened beyond detection by the paramagnetic effect of $\mathrm{Co}(\mathrm{II})$. Perturbations were calculated using $\Delta \delta=\left[\left(\Delta p p m{ }^{1} \mathrm{H}\right)^{2}+\left(\Delta p p m{ }^{15} \mathrm{~N} / 7\right)^{2}\right]^{1 / 2}$ 


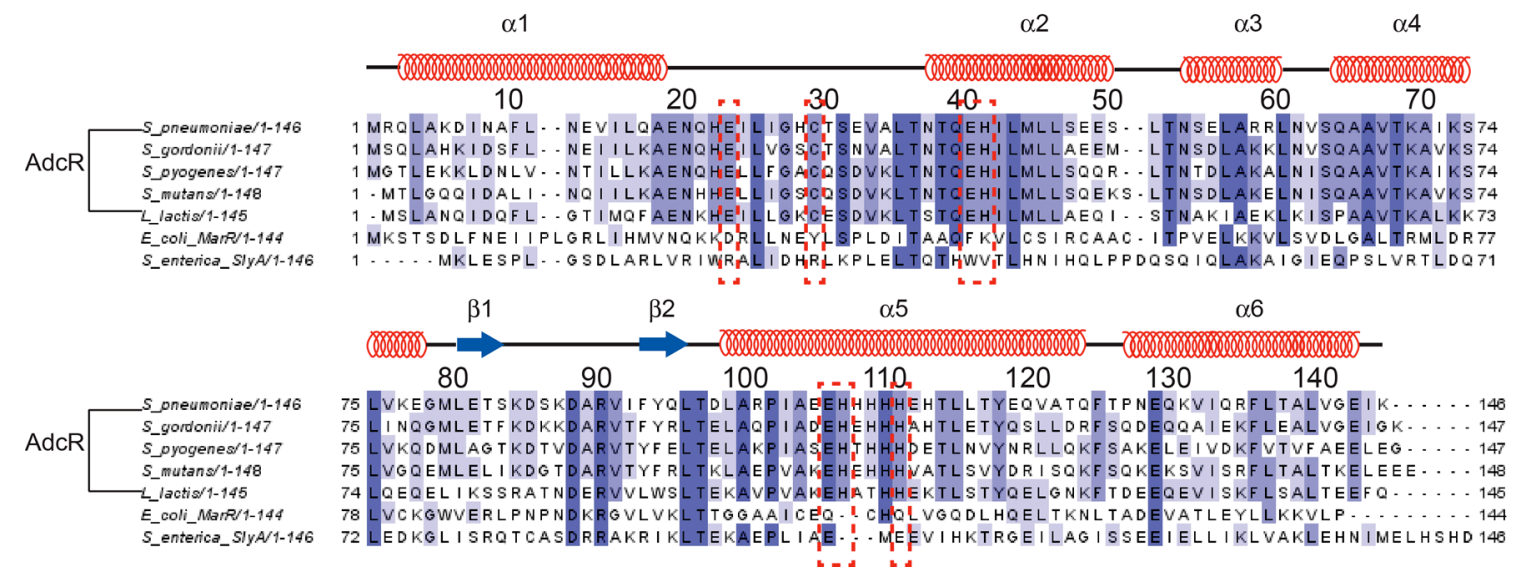

Figure S6. Primary structure alignment of S. pneumoniae AdcR with putative AdcRs from S. gordonii, S. pyogenes and S. mutans; L. lactis ZitR; ${ }^{11}$ as well as two structurally characterized MarR family members, E. coli MarR and S. enterica SlyA. ${ }^{12,13}$ The alignment was made using ClustalW and colored by percent identity using Jalview ${ }^{14,15}$. Secondary structure elements of $\mathrm{Zn}$ (II)-AdcR are indicated above the alignment as defined by the crystal structure and the metal binding residues are enclosed in red dashed boxes. 

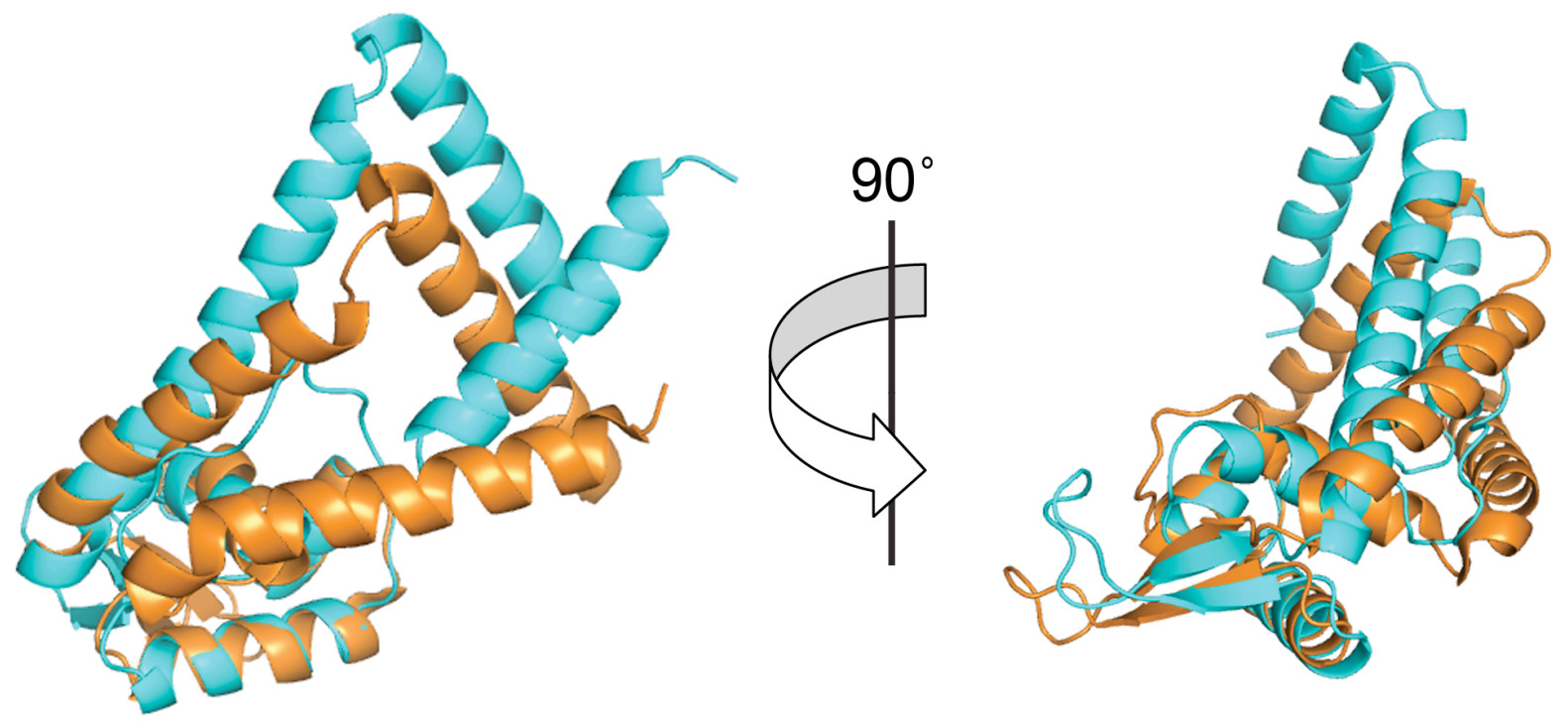

Figure S7. Superimposition of the winged helical domains of chain B of $\mathrm{Zn}(\mathrm{II})$-AdcR and one protomer of SlyA in the DNA-bound form. AdcR chain B is colored cyan and the SlyA protomer is colored orange. The DNA has been omitted for clarity. 


\section{Supplementary References}

Vol. 1.

(1) Doublié, S. Macromolecular crystallography protocols; Humana Press Inc.: Totowa;

(2) Reyes-Caballero, H.; Guerra, A. J.; Jacobsen, F. E.; Kazmierczak, K. M.; Cowart, D.; Koppolu, U. M.; Scott, R. A.; Winkler, M. E.; Giedroc, D. P. J. Mol. Biol. 2010, 403, 197.

(3) Arunkumar, A. I.; Pennella, M. A.; Kong, X.; Giedroc, D. P. Biomol NMR Assign 2007, 1,99 .

(4) Tugarinov, V.; Kay, L. E. J. Am. Chem. Soc. 2003, 125, 13868.

(5) Delaglio, F.; Grzesiek, S.; Vuister, G. W.; Zhu, G.; Pfeifer, J.; Bax, A. J. Biomol. NMR $1995,6,277$.

(6) Goddard, T. D.; Kneller, D. G.; University of California, San Francisco.

(7) Wishart, D. S.; Sykes, B. D. J. Biomol. NMR 1994, 4, 171.

(8) Otwinowski, Z. In Proceedings of the CCP4 study weekend: data collection and processing; Sawyer, L., Isaacs, N., Bailey, S., Eds.; SERC Daresbury Laboratory: Warrington, UK, 1993 , p 56.

(9) Adams, P. D.; Afonine, P. V.; Bunkoczi, G.; Chen, V. B.; Davis, I. W.; Echols, N.; Headd, J. J.; Hung, L.-W.; Kapral, G. J.; Grosse-Kunstleve, R. W.; McCoy, A. J.; Moriarty, N. W.; Oeffner, R.; Read, R. J.; Richardson, D. C.; Richardson, J. S.; Terwilliger, T. C.; Zwart, P. H. Acta Crystallogr. Sect. D. Biol. Crystallogr. 2010, 66, 213.

(10) Emsley, P.; Cowtan, K. Acta Crystallogr. Sect. D. Biol. Crystallogr. 2004, 60, 2126.

(11) Llull, D.; Son, O.; Blanie, S.; Briffotaux, J.; Morello, E.; Rogniaux, H.; Danot, O.; Poquet, I. J. Bacteriol. 2011, 193, 1919.

(12) Alekshun, M. N.; Levy, S. B.; Mealy, T. R.; Seaton, B. A.; Head, J. F. Nat. Struct. Biol. 2001, 8,710 .

(13) Dolan, K. T.; Duguid, E. M.; He, C. J. Biol. Chem. 2011, 286, 22178.

(14) Thompson, J. D.; Higgins, D. G.; Gibson, T. J. Nucleic Acids Res. 1994, 22, 4673.

(15) Waterhouse, A. M.; Procter, J. B.; Martin, D. M.; Clamp, M.; Barton, G. J. Bioinformatics 2009, 25, 1189. 